\title{
Research progress and prospects of complete ammonia oxidizing bacteria in wastewater treatment
}

\author{
Shaoping Luo ${ }^{1}$, Yi Peng ${ }^{2}$, Ying Liu ${ }^{3}$, Yongzhen Peng $(\bowtie){ }^{1}$ \\ 1 National Engineering Laboratory for Advanced Municipal Wastewater Treatment and Reuse Technology, Engineering Research Center of Beijing, \\ Beijing University of Technology, Beijing 100124, China \\ 2 Xinkai Water Environmental Investment Co. Ltd., Beijing 101101, China \\ 3 Zhongshan Public Utilities Water Co. Ltd., Zhongshan 528400, China
}

\section{H I G H L I G H T S}

- Comammox bacteria have unique physiological characteristics.

- Comammox bacteria are widely distributed in natural and artificial systems.

- Comammox bacteria have the potential to reduce $\mathrm{N}_{2} \mathrm{O}$ emissions.

- Coupling comammox bacteria with DEAMOX can be promoted in wastewater treatment.

- Comammox bacteria have significant potential for enhancing total nitrogen removal.

\section{A R T I C LE I N F O}

Article history:

Received 22 September 2021

Revised 14 December 2021

Accepted 27 December 2021

Available online 18 February 2022

Keywords:

Complete ammonia oxidizing (comammox) bacteria

Nitrogen cycle

Physiological characteristics

Wastewater treatment

\section{GRAPHIC ABSTRACT}

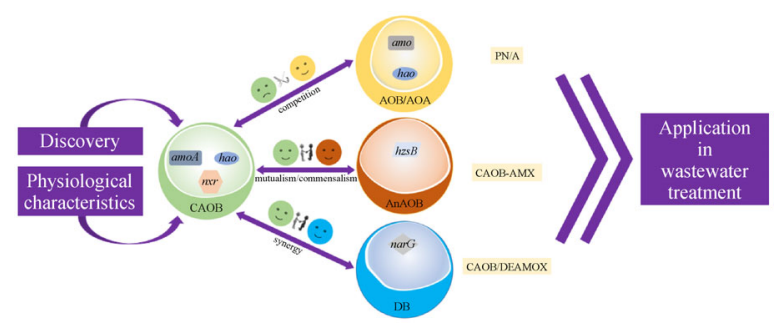

\section{A B S T R A C T}

Complete ammonia oxidizing bacteria, or comammox bacteria (CAOB), can oxidize ammonium to nitrate on its own. Its discovery revolutionized our understanding of biological nitrification, and its distribution in both natural and artificial systems has enabled a reevaluation of the relative contribution of microorganisms to the nitrogen cycle. Its wide distribution, adaptation to oligotrophic medium, and diverse metabolic pathways, means extensive research on $\mathrm{CAOB}$ and its application in water treatment can be promoted. Furthermore, the energy-saving characteristics of high oxygen affinity and low sludge production may also become frontier directions for wastewater treatment. This paper provides an overview of the discovery and environmental distribution of $\mathrm{CAOB}$, as well as the physiological characteristics of the microorganisms, such as nutrient medium, environmental factors, enzymes, and metabolism, focusing on future research and the application of CAOB in wastewater treatment. Further research should be carried out on the physiological characteristics of $\mathrm{CAOB}$, to analyze its ecological niche and impact factors, and explore its application potential in wastewater treatment nitrogen cycle improvement.

(C) The Author(s) 2022. This article is published with open access at link.springer.com and journal.hep. com.cn

\section{Introduction}

As a key process in the natural nitrogen cycle, nitrification is a microbial process by which ammonium $\left(\mathrm{NH}_{4}{ }^{+}-\mathrm{N}\right)$ is sequentially oxidized to nitrite $\left(\mathrm{NO}_{2}^{-}-\mathrm{N}\right)$ and nitrate $\left(\mathrm{NO}_{3}{ }^{-}\right.$ -N) by two separate organisms (Winogradsky, 1890;

$\triangle$ Corresponding author

E-mail: pyz@bjut.edu.cn

Special Issue-Visions
Kuypers et al., 2018). The first step is ammonia oxidation $\left(\mathrm{NH}_{4}{ }^{+}-\mathrm{N} \rightarrow \mathrm{NO}_{2}^{-}-\mathrm{N}\right.$, Eq. 1), mediated by ammonia oxidizing microorganisms (AOM), including ammonia oxidizing bacteria (AOB) and ammonia oxidizing archaea (AOA), which use ammonia monooxygenase (AMO) and hydroxylamine dehydrogenase (HAO) to oxidize ammonia to nitrite (Arp et al., 2002; Könneke et al., 2005). The second step is nitrite oxidation $\left(\mathrm{NO}_{2}{ }^{-} \mathrm{N} \rightarrow \mathrm{NO}_{3}{ }^{-} \mathrm{N}\right.$, Eq. 2), catalyzed by nitrite oxidizing bacteria (NOB), which uses nitrite oxidoreductase (NXR) to oxidize nitrite to nitrate (Teske et al., 1994). However, in 2015, the 
discovery of complete ammonia oxidizing bacteria, which can oxidize ammonium directly to nitrate in a single organism (Fig. 1), challenged our understanding of the conventional two-step nitrification process (Daims et al., 2015; Van Kessel et al., 2015). Furthermore, comammox bacteria have been detected in various environments, such as wastewater treatment facilities, drinking water systems, natural wetlands, lake sediments, and soil (Wang et al., 2017; Annavajhala et al., 2018; Xia et al., 2018). Their wide distribution has attracted great interest in their ecological role compared with other nitrifiers.

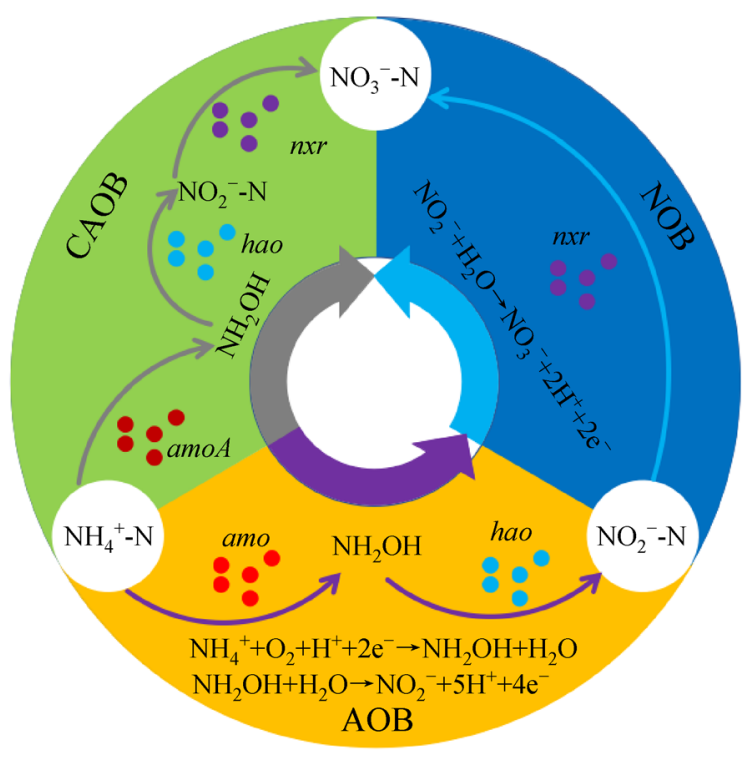

Fig. 1 Differences between traditional microbial nitrification and comammox bacteria nitrification.

After comammox bacteria were discovered, comparisons of comammox bacteria with the amo $A$ gene in the NCBI database identified a large number of highly similar sequences, in both natural (forests, plains, wetlands, etc.) and artificial systems (drinking water plants, sewage treatment plants, paddy fields, etc.) (Erwin et al., 2005; Knief et al., 2006; Jia et al., 2007; Vigliotta et al., 2007; Steenbergh et al., 2010; Daims et al., 2015; Van Kessel et al., 2015). Later research confirmed this, and found significant differences in abundance between habits (Xia et al., 2018). The distribution difference of clades A and B had a certain relationship with the difference of the encoded genome (Palomo et al., 2018; Koch et al., 2019).

Comammox bacteria have recently been identified in water treatment systems, but their contribution to biological nitrogen removal remains unclear (Daims et al., 2015; Van Kessel et al., 2015; Pjevac et al., 2017; Wang et al., 2017; Zhao et al., 2019). They significantly contribute to nitrification in water treatment systems (Pinto et al., 2015; Xia et al., 2018), with the abundance ratio of comammox bacteria to total amoA genes in some wastewater treatment plants reaching up to $100 \%$ (Zhou et al., 2021). How to utilize the physiological characteristics of comammox bacteria in aquatic ecosystems is of great significance. The integration of comammox bacteria into existing wastewater treatment processes has important research prospects and application potential for improving the effectiveness and stability of domestic wastewater treatment, reducing sewage treatment costs, and decreasing greenhouse gas emissions.

\section{Discovery of comammox bacteria}

Costa et al. (2006) speculated that, from a thermodynamic perspective, there was a microorganism that can completely oxidize ammonium to nitrate $\left(\mathrm{NH}_{4}{ }^{+}-\mathrm{N} \rightarrow \mathrm{NO}_{3}{ }^{-}-\mathrm{N}\right.$, Eq. 3). This single microorganism, which can complete nitrification, is named complete ammonia oxidizing bacteria (CAOB). In 2015, two laboratories in the Netherlands and Austria found strains in the genus Nitrospira that can independently complete the entire nitrification process, confirming the existence of comammox bacteria (Daims et al., 2015; Van Kessel et al., 2015).

$$
\begin{gathered}
\mathrm{NH}_{4}^{+}+1.5 \mathrm{O}_{2} \rightarrow \mathrm{NO}_{2}^{-}+\mathrm{H}_{2} \mathrm{O}+2 \mathrm{H}^{+} \\
(\Delta \mathrm{G}=-274.7 \mathrm{KJ} / \mathrm{mol}) \\
\mathrm{NO}_{2}^{-}+0.5 \mathrm{O}_{2} \rightarrow \mathrm{NO}_{3}^{-}(\Delta \mathrm{G}=-74.1 \mathrm{KJ} / \mathrm{mol}) \\
\mathrm{NH}_{4}^{+}+2 \mathrm{O}_{2} \rightarrow \mathrm{NO}_{3}^{-}+\mathrm{H}_{2} \mathrm{O}+2 \mathrm{H}^{+} \\
(\Delta \mathrm{G}=-348.9 \mathrm{KJ} / \mathrm{mol})
\end{gathered}
$$

\subsection{Candidatus Nitrospira inopinata}

Daims et al. (2015) collected biofilm from the wall of a hot water pipe of a 1200 m deep oil exploration well in North Caucasus, Russia, and enriched it in an ammoniumcontaining inorganic medium at a constant temperature of $46{ }^{\circ} \mathrm{C}$. The enriched culture could oxidize $\mathrm{NH}_{4}^{+}-\mathrm{N}$ to $\mathrm{NO}_{3}^{-}-\mathrm{N}$. By detecting the specific rRNA targeting probe of nitrifying bacteria, fluorescent in situ hybridization (FISH) confirmed that the enriched culture only contained Nitrospira, and the rest were Betaproteobacterium without ammonia oxidation ability. A polymerase chain reaction (PCR) did not detect any known amoA genes or 16S rRNA genes of AOA and AOB. Metagenomic sequencing found that the relative abundance of Nitrospira in the enriched culture was $68 \%-80 \%$. Further enrichment revealed that Nitrospira could oxidize $\mathrm{NH}_{4}{ }^{+}-\mathrm{N}$ to $\mathrm{NO}_{3}{ }^{-}-\mathrm{N}$. Therefore, it was considered to be a microbial strain with complete ammonia oxidation ability and was named Candidatus Nitrospira inopinata (Ca. N. inopinata). In 2017, Kits et al. 
(2017) obtained the pure culture strain of $C a$. N. inopinata by enrichment culture, which was also the first pure culture strain of comammox bacteria.

\subsection{Candidatus Nitrospira nitrosa and Candidatus Nitrospira nitrificans}

Also in 2015, van Kessel et al. (2015) sampled the anaerobic compartment of a trickling filter connected to a recirculation aquaculture system. Under low dissolved oxygen $\left(\mathrm{DO} \leqslant 3.1 \mu \mathrm{mol} / \mathrm{L} \mathrm{O}_{2}\right.$ ) and oligotrophic conditions, the genus Brocadia of anammox bacteria $(\mathrm{AnAOB})$ and two different Nitrospira genus were enriched by culturing for 12 months. A high abundance of Nitrospira was rarely seen under low DO conditions. Both NOB species carried the genes (amo and hao) for ammonia oxidation as well as the $n x r$ gene necessary for nitrite oxidation; therefore, these two strains were confirmed to have the metabolic potential to oxidize $\mathrm{NH}_{4}{ }^{-}-\mathrm{N}$ to $\mathrm{NO}_{3}{ }^{-}-\mathrm{N}$, and are named Candidatus Nitrospira nitrosa (Ca. N. nitrosa) and Candidatus Nitrospira nitrificans ( $\mathrm{Ca}$. N. nitrificans), respectively. The phylogeny of $16 \mathrm{~S}$ rRNA genes indicates that they belong to lineage II of the genus Nitrospira.

\subsection{Candidatus Nitrospira kreftii}

Sakoula et al. (2021) used a continuous membrane bioreactor with an effective volume of $5 \mathrm{~L}$ containing Ca. N. nitrosa and Ca. N. nitrificans (van Kessel et al., 2015), operated at room temperature and under suitable conditions (e.g., $\mathrm{pH}=7.5, \mathrm{DO}=50 \%$ oxygen saturation) for 39 months. They obtained a novel comammox bacteria species, Candidatus Nitrospira kreftti (Ca. N. kreftii), which displayed clear differences in terms of ammonia and nitrite oxidation kinetics. For example, $\mathrm{Ca}$. N. kreftti has a higher nitrite affinity. Furthermore, partial inhibition of ammonia oxidation at ammonium concentrations as low as $25 \mu \mathrm{M}$ was observed in the bioreactor, indicating that differences in ammonium tolerance could potentially be a niche-determining factor for different comammox Nitrospira.

\subsection{Summary}

All identified comammox are Nitrospira bacteria, belonging to lineage II of the Nitrospira genus, and are phylogenetically divided into two branches according to the amoA gene: clade A and clade B. These two clades have significant differences in substrate operation and metabolism, energy transfer, and environmental adaptability (Palomo et al., 2018). In comammox bacteria enrichment cultures, genome analysis has identified the amo A gene, encoding AMO; hao encoding HAO; and $n x r$ encoding NXR, which can independently perform the complete nitrification process (Daims et al., 2015; Van Kessel et al., 2015; Sakoula et al., 2021). Van Kessel et al.
(2015) found that the amoA gene in $C a$. N. nitrosa and $C a$. $N$. nitrificans is different from that of typical ammonia oxidizing bacteria, and belongs to two different branches phylogenetic, but is highly similar to pmoA of the methane monooxygenase of Crenohrix polyspora. Therefore, it is inferred that the amo $A$ gene of some comammox bacteria in the previous study may be incorrectly classified as methane monooxygenase and could be ignored (Daims et al., 2015; Van Kessel et al., 2015).

The discovery of comammox bacteria revolutionized our understanding of the nitrification process, and the relative contribution of microorganisms to the nitrogen cycle should be reassessed.

\section{Physiological characteristics of comammox bacteria}

\subsection{Nutrient matrix}

Comammox bacteria have advantages over traditional nitrifying bacteria in oligotrophic substrates, such as low ammonia nitrogen and low DO (Kits et al., 2017). In addition to the reported AOA of marine $N$. maritimus $S C M 1$, the ammonia half-saturation constant of AOA and AOB is much higher than that of purified and cultured $\mathrm{Ca}$. $N$. inopinata, but the affinity of comammox bacteria to nitrite is much lower than that of traditional NOB (Kits et al., 2017). Zhao et al. (2021) used urea and nitrite to enrich and cultivate comammox bacteria for 390 days to increase the ratio of amo $A$ gene, indicating that comammox bacteria have diverse metabolic potential.

\subsection{Environmental factors}

Environmental conditions, such as $\mathrm{DO}, \mathrm{pH}$, and temperature have a significant impact on the growth and activity of comammox bacteria (Kits et al.,2017; Palomo et al., 2018; Roots et al., 2019; Takahashi et al., 2020; Sun et al., 2020). The microbial oxidase of comammox bacteria is similar to cytochrome bd-type terminal oxidase, which is generally expressed under extremely low DO concentrations and has a higher affinity for DO (Borisov et al., 2011). There are currently no reports on the half-saturation constant of oxygen, but comammox bacteria are more competitive under low DO conditions. With increasing DO, AOA and AOB activities gradually increase, but comammox bacteria lose their competitiveness (Palomo et al., 2018; Roots et al., 2019). Sun et al. (2020) found that when the $\mathrm{pH}$ was between 6.74 and 8.65 , the abundance of comammox bacteria in coastal wetlands was positively correlated with $\mathrm{pH}$. In addition, comammox bacteria can show nitrifying activity at $\mathrm{pH} 3-4$, indicating that comammox bacteria may be able to adapt to low pH (Takahashi et al., 2020). Shi et al. found that the abundance of comammox bacteria was relatively high in samples collected from wetland ecosys- 
tems. Based on the changes in comammox bacteria species diversity in summer and winter, they speculated that temperature may be a key factor affecting the structure of comammox bacteria (Shi et al., 2020). Zhou et al. (2021) tested the abundance, activity, and metabolic characteristics of nitrifying bacteria in drinking water and wastewater treatment plants with seasonal changes and found that the network complex of comammox microorganisms in sewage treatment plants was highest in December. However, a study by Kits et al. (2017) found that the optimal growth of $\mathrm{Ca}$. N. inopinata occurred at $37^{\circ} \mathrm{C}$, and the optimal enrichment culture temperature was $46{ }^{\circ} \mathrm{C}$. This indicates that different comammox bacteria can adapt to growth environments of different temperatures.

\subsection{Enzymes and metabolism}

The comammox bacteria metabolism pathway is complex and can adapt to different nutrient substrates (Daims et al., 2015; Van Kessel et al., 2015; Shen et al., 2016; Kits et al., 2017; Han et al., 2019; Liu et al., 2020a; Wang et al., 2021; Zhao et al., 2021). Liu et al. (2020a) found that comammox bacteria contribute $34 \%-87 \%$ of nitrification in a typical oligotrophic environment with high $\mathrm{pH}$ and low temperature along the Yangtze River. Kits et al. (2017) enriched and cultivated comammox bacteria under higher temperature conditions, demonstrating that comammox microorganisms can adapt to varying and more extreme environments. Metagenomic analysis shows that the discovered comammox bacteria can use urea as a nitrogen source for energy metabolism and biosynthesis (Daims et al., 2015; Van Kessel et al., 2015; Zhao et al., 2021). Studies have found that comammox bacteria can use hydrogen sulfide, sulfide, and fermentation products for metabolism (Kits et al., 2017), while trace organic pollutants in the environment can be removed through metabolism (Han et al., 2019). Some comammox may also have the potential to oxidize formic acid and hydrogen (Palomo et al., 2018), and a variety of antibiotics have been used to effectively and selectively enrich different strains of comammox bacteria (Wang et al., 2021). In addition, comammox bacteria have a gene encoding alkaline phosphatase, which can make it more advantageous under insufficient phosphorus conditions (Shen et al., 2016).

Comammox bacteria have a complete genome of key functional enzymes in the oxidation process of $\mathrm{NH}_{4}^{+}-\mathrm{N}$ to $\mathrm{NO}_{3}^{-}-\mathrm{N}$, and can complete the whole process of traditional two-step nitrification metabolism itself. The comammox bacteria genome encodes the nitrite reductase gene (nir) but lacks the nitric oxide reductase gene (nor). In theory, it produces $\mathrm{NO}$ but not $\mathrm{N}_{2} \mathrm{O}$ during biological reactions (Kits et al., 2019). Palomo et al. (2018) found that there was no pathway related to $\mathrm{NO}_{x}$ metabolism in the known comammox bacteria genome, and the $\mathrm{NO}_{x}$ reduction reaction may not be possible. Comammox bacteria sequester carbon through the reduced tricarboxylic acid (rTCA) cycle. The rTCA cycle is usually the mechanism of $\mathrm{CO}_{2}$ fixation by anaerobic or aerobic microorganisms (Lücker et al., 2010); compared with the Calvin cycle corresponding to $\mathrm{AOB}$, the fixed unit $\mathrm{CO}_{2}$ of the rTCA cycle consumes less adenosine triphosphate (ATP) and requires less oxygen (Lawson and Lücker, 2018). Theoretically, the comammox bacteria oxidation unit $\mathrm{NH}_{4}{ }^{+} \mathrm{N}$ has a shorter metabolic pathway and a higher growth yield than $\mathrm{AOB}$, so it is easier to obtain a competitive advantage under low DO conditions (Costa et al., 2006).

\subsection{Natural habitat distribution}

Similar to AOA, comammox bacteria have a high affinity for $\mathrm{NH}_{4}^{+}-\mathrm{N}$ and are suitable for growth under low $\mathrm{NH}_{4}^{+}-\mathrm{N}$ conditions. There are numerous natural systems suitable for the growth of comammox bacteria. Table 1 shows partial statistics of the distribution of comammox bacteria in natural ecosystems.

Comammox bacteria are widely distributed in nature, and the distribution further confirms that comammox bacteria like to grow under conditions of low DO and $\mathrm{NH}_{4}^{+}-\mathrm{N}$. AOA was first discovered in the marine environment. However, although the physiological characteristics of comammox bacteria are similar to those of AOA, Liu et al. (2020b) detected a high abundance of comammox bacteria in extremely saline sediments, showing that comammox bacteria can adapt to high-salinity environments, and a distinct differentiation of comammox Nitrospira groups driven by salinity has been identified (Sun et al. 2021). However, so far, comammox bacteria have not been found in the ocean (Daims et al., 2015; Kuypers, 2017; Xu et al. 2020), indicating that salinity is a key factor. Whether there are comammox bacteria in marine habitats is an important research direction.

At present, data on the nitrification kinetics of comammox bacteria are derived from pure cultured $\mathrm{Ca}$. $\mathrm{N}$. inopinata. (Kits et al., 2017). Therefore, there are some limitations for studying the nitrification kinetics of comammox. With the widespread discovery of comammox bacteria and the versatility of metabolism, research on the physiological characteristics of comammox bacteria will further understanding of the niche characteristics of comammox bacteria in the biosphere and its relative contribution to the nitrogen cycle.

\section{Application in wastewater treatment}

\subsection{Distribution in water systems}

In conventional artificial system studies, only the nitrification activity of $\mathrm{AOM}$ and $\mathrm{NOB}$ is evaluated, and the presence of comammox bacteria is rarely considered. NOB 
Table 1 Comammox bacteria partial distribution in natural ecosystems

\begin{tabular}{|c|c|c|c|c|c|}
\hline Growth environment & Country & Strains & Research method & Key finding & Reference \\
\hline $\begin{array}{l}\text { Atmospheric fine } \\
\text { particles }\end{array}$ & China & N. inopinata & $\begin{array}{c}\mathrm{PCR}^{\mathrm{a})} \\
\text { Clone sequencing }\end{array}$ & $\begin{array}{l}\text { The abundance of comammox bacteria in } \\
\text { the atmosphere is less than AOA and } \\
\text { greater than AOB. }\end{array}$ & Gao et al., 2016 \\
\hline $\begin{array}{l}\text { Forest soil, lake } \\
\text { sediments, freshwater } \\
\text { organisms, etc. }\end{array}$ & $\begin{array}{c}\text { Austria; } \\
\text { Netherlands }\end{array}$ & / & Metagenome & $\begin{array}{c}\text { A PCR primer set was developed } \\
\text { specifically for the subunit amoA gene } \\
\text { encoding the unique amo } A \text { of comammox } \\
\text { bacteria. }\end{array}$ & Pjevac et al., 2017 \\
\hline Forest soil & China & / & qPCR & $\begin{array}{l}\text { The abundance of comammox bacteria is } \\
\text { very high. When the } \mathrm{pH} \text { is } 4.0-9.0 \text {, the } \\
\text { abundance exceeds AOB and NOB. }\end{array}$ & $\mathrm{Hu}$ and $\mathrm{He}, 2017$ \\
\hline $\begin{array}{l}\text { Sediments from the } \\
\text { Yangtze River Estuary }\end{array}$ & China & $\begin{array}{l}\text { N. inopinata } \\
\text { N. nitrosa } \\
\text { N. nitrificans }\end{array}$ & $\begin{array}{c}\text { Metagenome; } \\
\text { Macrotranscriptome; } \\
\text { qPCR }\end{array}$ & $\begin{array}{l}\text { The presence of comammox bacteria was } \\
\text { detected, and a primer set for clade A was } \\
\text { designed to quantitatively detect the } a m o A \\
\text { gene of comammox bacteria. }\end{array}$ & Yu et al., 2018 \\
\hline Riparian soil & China & $\begin{array}{c}N . \text { nitrosa } \\
N . \text { nitrificans }\end{array}$ & $\begin{array}{c}\text { qPCR; } \\
\text { Correlation analysis }\end{array}$ & $\begin{array}{l}\text { The microbial abundance of comammox } \\
\text { in the riparian soil was } 10^{8} \text { copies } / \mathrm{g} \text {, and } \\
\text { the abundance of comammox and Nitrospira } \\
\text { was significantly correlated under certain } \\
\text { conditions. }\end{array}$ & Wang et al, 2019 \\
\hline
\end{tabular}

Note: a) qPCR: quantitative polymerase chain reaction.

of the Nitrospira genus was mistakenly thought to exist widely in artificial systems. Metagenomic detection and analysis show that some of these have a full set of characteristic genes for ammonia oxidation, indicating that they may be comammox bacteria (Pinto et al., 2015). Table 2 shows partial statistics on the distribution of comammox bacteria in artificial systems.

Comammox bacteria are widely distributed in natural and artificial systems (Fig. 2). However, previous calculations on the contribution of ammonia oxidizing microorganisms in the nitrogen cycle ignored the role of comammox bacteria. Theoretically, the $\mathrm{AOM} / \mathrm{NOB}$ value in the traditional nitrification process should be between two and three (Winkler et al., 2012), but studies have found that the abundance of Nitrospira in the same habitat exceeds AOM (Zeng et al., 2015), which cannot be explained by traditional nitrification theories. It has now been demonstrated that some Nitrospira may be comammox bacteria (Pinto et al., 2015; Tatari et al., 2017). The discovery of comammox will cause a reevaluation of the relative contribution of nitrification in the nitrogen cycle, and the structure of the flora.

\subsection{Potential application in wastewater treatment}

Wang et al. (2018) found that, among eight wastewater treatment plant samples investigated, activity abundance of amoA of the comammox bacteria in six samples was significantly higher than or comparable to that of AOB, even reaching 24 times more in some wastewater plants. The abundance of comammox bacteria to total amoA genes in drinking water plants and AAO wastewater treatment plants is up to $46 \%-100 \%$ (Zhou et al., 2021), indicating that comammox bacteria may play an important role in wastewater treatment. Sato et al. (2021) used a segmented reactor to first ammonify organic nitrogen, and then nitrate into nitrate as a hydroponic fertilizer for plant growth. Through 16S rRNA gene sequencing and shotgun metagenomic analysis, the dominant microorganism in the nitrification process was confirmed as comammox bacteria, and qPCR analysis showed that the expression of comammox-type AMO was more than 500 times higher than that of traditional AMO. Heise et al. (2021) found that comammox bacteria participated in the removal of $\mathrm{NH}_{4}^{+}-\mathrm{N}$ at low steady-state $\mathrm{NH}_{4}{ }^{+}-\mathrm{N}$ concentrations in an aquaponic system, indicating that comammox plays an important role in this system.

Research on the growth mechanisms and physiological characteristics of comammox bacteria can improve the treatment of urban domestic wastewater. Fig. 3 shows a common microbial denitrification process in wastewater plants. For urban wastewater partial nitrification-denitrification (PN/D) and partial nitrification-anaerobic ammonia oxidation (PN/A) denitrification systems, the growth and enrichment of comammox bacteria are one of the important reasons for the destruction of these systems (Wang et al., 2020). Controlling the growth of comammox bacteria is an important factor in realizing PN/D or PN/A of urban domestic wastewater. Purified and cultured comammox bacteria have a low affinity for nitrite (Kits et al., 2017; Xia et al., 2018). Meanwhile, comammox bacteria and anammox bacteria can form copolymers under hypoxic conditions (Van Kessel et al., 2015; Gottshall et al., 2021; Shao and $\mathrm{Wu}, 2021)$. In theory, the nitrite oxidation activity of comammox bacteria can be inhibited by controlling conditions to achieve stable PN/A. In the PN/ 
Table 2 Comammox bacteria partial distribution in artificial systems

\begin{tabular}{|c|c|c|c|c|c|}
\hline Growth environment & Country & Species & Research method & Key finding & Reference \\
\hline $\begin{array}{l}\text { Wastewater treatment } \\
\text { plants (WWTPs) }\end{array}$ & China & N. inopinata & $\begin{array}{l}\text { Metagenome; } \\
\text { 16S rRNA }\end{array}$ & $\begin{array}{c}\text { Comammox bacteria abundance accounts } \\
\text { for } \leqslant 0.1 \% \text {. It is speculated that the contribution } \\
\text { of comammox bacteria nitrification is small in the } \\
\text { sewage treatment process. }\end{array}$ & Chao et al., 2016 \\
\hline $\begin{array}{l}\text { Drinking water } \\
\text { systems }\end{array}$ & $\begin{array}{l}\text { Singapore; } \\
\text { China; } \\
\text { United States }\end{array}$ & $\begin{array}{l}\text { N. inopinata } \\
\text { N. nitrosa } \\
\text { N. nitrificans }\end{array}$ & Metagenome & $\begin{array}{l}\text { Comammox bacteria are widely distributed in } \\
\text { drinking water systems and coexist with traditional } \\
\text { AOM. The nitrification of drinking water systems } \\
\text { may be mainly completed by comammox bacteria. }\end{array}$ & Wang et al., 2017 \\
\hline WWTPs & United States & N. nitrosa & Metagenome & $\begin{array}{l}\text { To achieve enrichment of } C a \text {. } N \text {. nitrosa, it has a } \\
\text { higher affinity for urea. }\end{array}$ & Kits et al., 2017 \\
\hline WWTPs & $\begin{array}{l}\text { United States } \\
\text { United Kingdom }\end{array}$ & $\begin{array}{l}\text { N. inopinata } \\
\text { N. nitrosa } \\
\text { N. nitrificans }\end{array}$ & $\begin{array}{l}\text { Metagenome; } \\
\text { qPCR }\end{array}$ & $\begin{array}{l}\text { A primer set and qPCR targeting clade A were } \\
\text { designed; comammox bacteria prefer long sludge age } \\
\text { and attached growth, and the increase in abundance } \\
\text { in the same habitat has no correlation with the } \\
\text { decrease in AOB and NOB abundance. }\end{array}$ & $\begin{array}{c}\text { Camejo et al., } \\
2017\end{array}$ \\
\hline Urban lake & China & / & 16S rRNA & $\begin{array}{l}\text { Comammox bacteria are widely distributed in } \\
\text { urban lakes, eutrophication may inhibit its growth. }\end{array}$ & Xu et al., 2020 \\
\hline Acidic soils & Japan & / & $16 \mathrm{~S}$ rRNA & $\begin{array}{l}\text { When the } \mathrm{pH} \text { is } 3-4 \text {, nitrification activity of } \\
\text { comammox bacteria is still detected. }\end{array}$ & $\begin{array}{c}\text { Takahashi et al., } \\
2020\end{array}$ \\
\hline $\begin{array}{l}\text { A continuous } \\
\text { membrane } \\
\text { bioreactor }\end{array}$ & Netherlands & N. Kreftii & $\begin{array}{c}\text { FISH; } \\
\text { Metagenome; }\end{array}$ & $\begin{array}{c}\text { They obtained a novel comammox bacteria species, } \\
\mathrm{Ca} \text {. N. kreftit. Moreover, they think differences in } \\
\text { ammonium tolerance could potentially be a } \\
\text { niche-determining factor for different comammox } \\
\text { Nitrospira. }\end{array}$ & $\begin{array}{l}\text { Sakoula et al., } \\
2021\end{array}$ \\
\hline $\begin{array}{l}\text { Lab-scale PN/A SBR } \\
\text { Reactor }\end{array}$ & China & / & $\begin{array}{l}\text { 16S rRNA } \\
\text { qPCR }\end{array}$ & $\begin{array}{l}\text { The amoA gene of comammox bacteria in the } \\
\text { PN/A system that has been running stably for more } \\
\text { than } 1,000 \text { days accounted for } 89.2 \pm 7.9 \% \text {, achieving } \\
\text { synergistic denitrification under hypoxic conditions. }\end{array}$ & $\begin{array}{l}\text { Shao and } \mathrm{Wu} \text {, } \\
2021\end{array}$ \\
\hline Aquaponic system & Germany & / & 16S rRNA & $\begin{array}{l}\text { Comammox bacteria are found in the high- } \\
\text { efficiency aquaponic symbiosis system, which } \\
\text { participate in the removal of } \mathrm{NH}_{4}^{+}-\mathrm{N} \text { at low } \\
\text { steady-state } \mathrm{NH}_{4}^{+}-\mathrm{N} \text { concentrations. }\end{array}$ & Heise et al., 2021 \\
\hline
\end{tabular}

A process, $\mathrm{NH}_{4}^{-}-\mathrm{N}$ and $\mathrm{DO}$ continuously decrease as particles or biofilms develop, which may lead to the enrichment of comammox bacteria (Wang et al., 2020), competing with $\mathrm{AOB}$ and reducing the total nitrogen removal rate, instead of the traditional NOB proliferation effect.

In some wastewater treatment plants, comammox bacteria account for a relatively high proportion of activated sludge (Zhou et al., 2021). An important research direction in wastewater treatment is how to use the physiological characteristics of comammox bacteria to further reduce sewage treatment costs and improve treatment effects. Using comammox bacteria to treat low $\mathrm{NH}_{4}^{+}-\mathrm{N}$ municipal sewage under low DO conditions can reduce the aeration; therefore, the energy consumption. The generation of sludge can also be reduced, as well as the cost of post-sludge treatment and disposal. With the recent research and development of partial denitrification $\left(\mathrm{NO}_{3}^{-}-\right.$ $\mathrm{N} \rightarrow \mathrm{NO}_{2}^{-}-\mathrm{N}$ ) and the anaerobic ammonia oxidation coupling process (DEAMOX), simultaneous treatment of $\mathrm{NO}_{3}^{-}-\mathrm{N}$ and $\mathrm{NH}_{4}^{+}-\mathrm{N}$ in the same reactor has been realized.
Compared with PN/A, the DEAMOX process has many advantages, such as a more stable accumulation of $\mathrm{NO}_{2}^{-}-\mathrm{N}$, no complicated control, and theoretical $100 \%$ deep denitrification. In this process, comammox bacteria are used to couple partial denitrification-anaerobic ammonia oxidation to develop a new type of denitrification process. Hypoxic nitrification reduces aeration energy consumption, and anoxic partial denitrification-anaerobic ammonia oxidation saves carbon sources (Fig. 4). The process is stable and easy to control, with a low sludge output and a reduction in greenhouse gas emissions, and may be more suitable for saving energy, reducing consumption, and deep denitrification (Xu et al., 2021).

Traditional biological denitrification is usually achieved through nitrification and denitrification, producing $\mathrm{N}_{2} \mathrm{O}$ in the process. $\mathrm{N}_{2} \mathrm{O}$ is an important greenhouse gas. Comammox bacteria have no $\mathrm{N}_{2} \mathrm{O}$ biosynthesis pathway; therefore, using comammox bacteria as the main reaction microorganism in the nitrification process may greatly reduce $\mathrm{N}_{2} \mathrm{O}$ emissions. Comammox bacteria can oxidize low ammonia nitrogen to nitrate nitrogen under low DO 


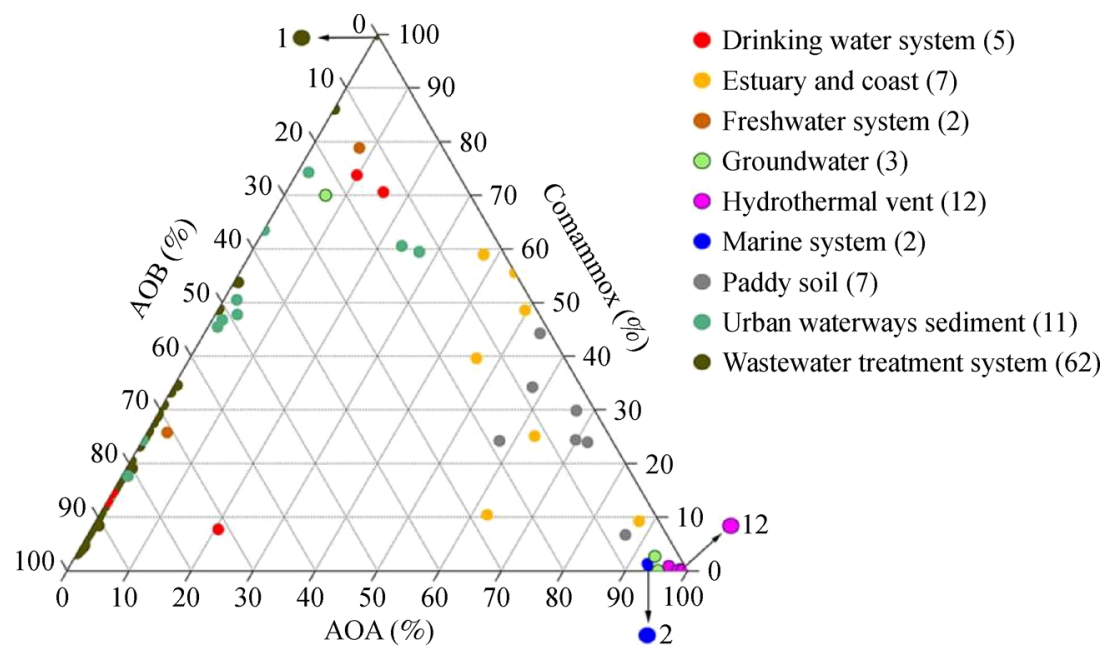

Fig. 2 Ternary plot of the proportions of comammox, AOB, and AOA in 111 metagenomic data sets of different environmental samples from the NCBI SRA database (Xia et al. 2018).

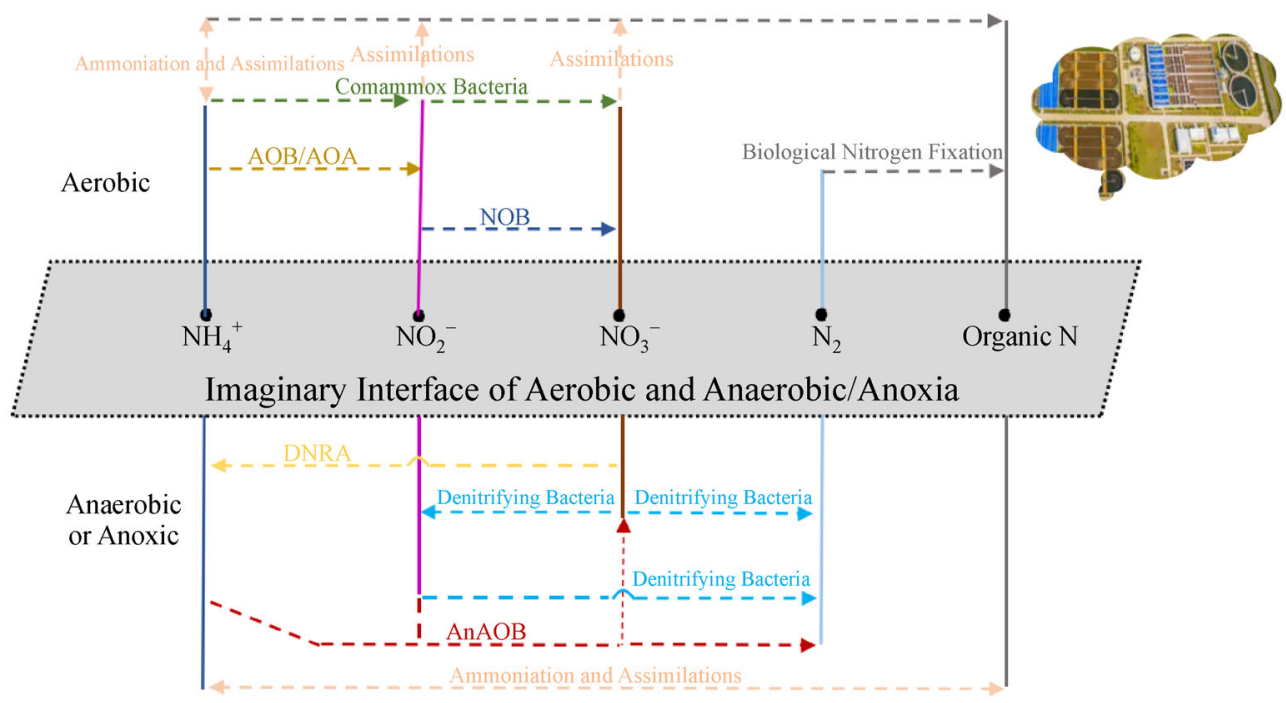

Fig. 3 Common microbial denitrification processes in wastewater treatment plants. (DNRA: dissimilatory nitrate reduction to ammonium).

conditions. Low DO meets the reaction conditions of simultaneous denitrification (Holman and Wareham, 2005). Simultaneous nitrification and denitrification can reduce internal circulation and aeration energy consumption and save space, while the nitrification process, avoiding the accumulation of nitrite, can be used to treat micro-polluted drinking water, improve treatment efficiency, and avoid the potential risk of nitrite production.

\section{Conclusions and outlook}

This review summarizes the discovery, physiological characteristics, and ecological distribution of comammox bacteria, and proposes potential applications in wastewater treatment. All comammox that have been discovered are Nitrospira bacteria, belonging to lineage II of the Nitrospira genus. The affinity of comammox bacteria to ammonium is higher and is also slower growing than conventional AOB/NOB, so better adapted to oligotrophic conditions. Furthermore, comammox bacteria have a complex pathway and can live in many adverse environments, as demonstrated by their wide distribution in natural and artificial systems. Coupling with DEAMOX or enriching in oligotrophic conditions can promote total nitrogen removal, thereby reducing both costs and $\mathrm{N}_{2} \mathrm{O}$ emissions.

The discovery of comammox bacteria has important significance and research value for calculating and evaluating the relative contribution of nitrification in the 


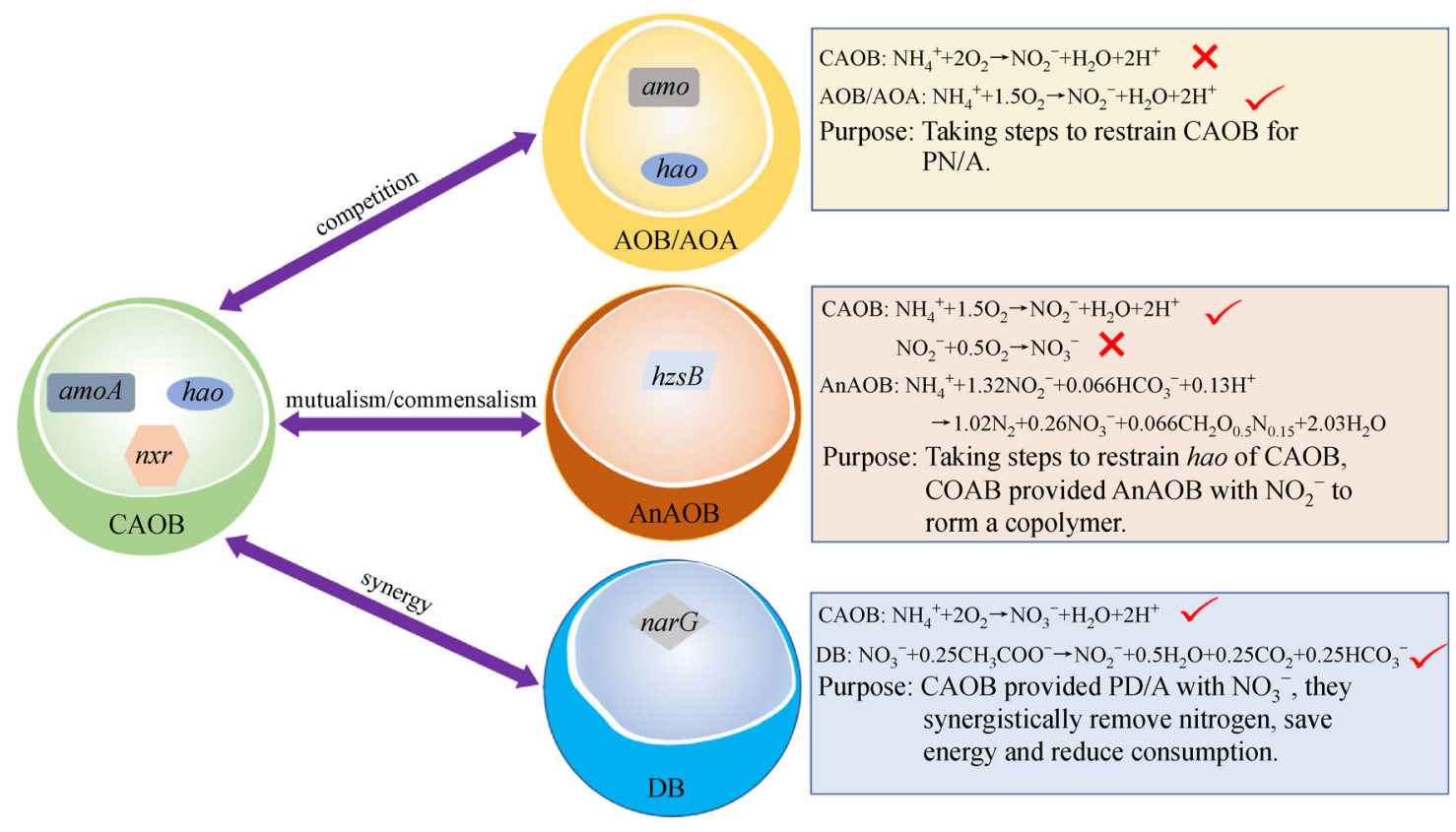

Fig. 4 Application of comammox bacteria in wastewater treatment. (DB: denitrifying bacteria).

microbial nitrogen cycle. To detect the presence and abundance of comammox bacteria using the amoA gene of comammox bacteria, a variety of specific primers have been developed to identify and quantify comammox bacteria of different branches and species levels. However, the accuracy and sensitivity of these need to be further improved.

Comammox bacteria are of great significance in both natural and artificial systems, but the niche differences and influencing factors between them and $\mathrm{AOB}, \mathrm{AOA}, \mathrm{NOB}$, and other microorganisms remain unclear, and may be a research hotspot for microbial applications in the future. At present, our understanding of comammox bacteria is limited, and the in situ activity of comammox bacteria cannot be fully evaluated. There is an urgent need to develop isolation and purification methods to study the physiological characteristics of microorganisms at the single-cell level, and to explore the coupling mechanism between comammox bacteria and traditional nitrification and denitrification. For the treatment of micro-polluted water sources, it is necessary to further explore the enrichment and operating conditions of comammox bacteria. In wastewater treatment, the use of comammox bacteria can reduce aeration energy consumption, greenhouse gas $\mathrm{N}_{2} \mathrm{O}$ emissions, and sludge production, while improving deep denitrification efficiency. It has a broad application potential and may become a new biological denitrification process in the future.

The following areas should be given priority in future studies on comammox bacteria: 1) Efficient and accurate methods that can identify the contribution of diverse microorganisms from similar functional microbes should be developed (e.g., AOB/AOA/NOB/comammox bacteria), and the accuracy and sensitivity of specific primers need to be further improved; 2) In wastewater treatment plants, the quantity of comammox bacteria as a proportion of nitrifying bacteria varies, and the relative contribution is not clear; evaluation and calculation of this should be strengthened in the future; 3 ) Comammox bacteria are used to couple DEAMOX to develop a new nitrogen removal process, the potential of comammox bacteria in more sustainable nitrogen removal techniques could be assessed from the point of view of engineering applications.

Acknowledgements This research project was financially supported by the Foundation for Innovation Research Groups of the National Natural Science Foundation of China (No. 62021003); Biological Wastewater Treatment and Process Control Technology, Beijing International Science and technology Cooperation Base; and the Founding projects of Beijing Municipal Commission of Education.

Open Access This article is licensed under a Creative Commons Attribution 4.0 International License, which permits use, sharing, adaptation, distribution and reproduction in any medium or format, as long as you give appropriate credit to the original author(s) and the source, provide a link to the Creative Commons licence, and indicate if changes were made. The images or other third party material in this article are included in the article's Creative Commons licence, unless indicated otherwise in a credit line to the material. If material is not included in the article's Creative Commons licence and your intended use is not permitted by statutory regulation or exceeds the permitted use, you will need to obtain permission directly from the copyright holder. To view a copy of this licence, visit http://creativecommons.org/licenses/by/4.0/.

\section{References}

Annavajhala M K, Kapoor V, Santo-Domingo J, Chandran K (2018). 
Comammox functionality identified in diverse engineered biological wastewater treatment systems. Environmental Science \& Technology Letters, 5(2): 110-116

Arp D J, Sayavedra-Soto L A, Hommes N G (2002). Molecular biology and biochemistry of ammonia oxidation by Nitrosomonas europaea. Archives of Microbiology, 178(4): 250-255

Borisov V B, Gennis R B, Hemp J, Verkhovsky M I (2011). The cytochrome bd respiratory oxygen reductases. Biochimica et Biophysica Acta (BBA) - Bioenergetics, 1807(11): 1398-1413

Camejo P Y, Santo Domingo J, McMahon K D, Noguera D R (2017). Genome-enabled insights into the ecophysiology of the comammox bacterium "Candidatus Nitrospira nitrosa". mSystems, 2(5): e00059-e17

Chao Y Q, Mao Y P, Yu K, Zhang T (2016). Novel nitrifiers and comammox in a full-scale hybrid biofilm and activated sludge reactor revealed by metagenomic approach. Applied Microbiology and Biotechnology, 100(18): 8225-8237

Costa E, Pérez J, Kreft J U (2006). Why is metabolic labour divided in nitrification? Trends in Microbiology, 14(5): 213-219

Daims H, Lebedeva E V, Pjevac P, Han P, Herbold C, Albertsen M, Jehmlich N, Palatinszky M, Vierheilig J, Bulaev A, Kirkegaard R H, von Bergen M, Rattei T, Bendinger B, Nielsen P H, Wagner M (2015). Complete nitrification by Nitrospira bacteria. Nature, 528 (7583): 504-509

Erwin D P, Erickson I K, Delwiche M E, Colwell F S, Strap J L, Crawford R L (2005). Diversity of oxygenase genes from methaneand ammonia-oxidizing bacteria in the Eastern Snake River Plain aquifer. Applied and Environmental Microbiology, 71(4): 2016-2025

Gao J F, Fan X Y, Pan K L, Li H Y, Sun L X (2016). Diversity, abundance and activity of ammonia-oxidizing microorganisms in fine particulate matter. Scientific Reports, 6(1): 38785

Gottshall E Y, Bryson S J, Cogert K I, Landreau M, Sedlacek C J, Stahl D A, Daims H, Winkler M (2021). Sustained nitrogen loss in a symbiotic association of comammox Nitrospira and anammox bacteria. Water Research, 202(1): 117426

Han P, Yu Y, Zhou L, Tian Z, Li Z, Hou L, Liu M, Wu Q, Wagner M, Men Y (2019). Specific micropollutant biotransformation pattern by the comammox bacterium Nitrospira inopinata. Environmental Science \& Technology, 53(15): 8695-8705

Heise J, Müller H, Probst A J, Meckenstock R U (2021). Ammonium removal in aquaponics indicates participation of comammox Nitrospira. Current Microbiology, 78(3): 894-903

Holman J B, Wareham D G (2005). COD, ammonia and dissolved oxygen time profiles in the simultaneous nitrification/denitrification process. Biochemical Engineering Journal, 22(2): 125-133

Hu H W, He J Z (2017). Comammox-A newly discovered nitrification process in the terrestrial nitrogen cycle. Journal of Soils and Sediments, 17(12): 2709-2717

Jia Z J, Kikuchi H, Watanabe T, Asakawa S, Kimura M (2007). Molecular identification of methane oxidizing bacteria in a Japanese rice field soil. Biology and Fertility of Soils, 44(1): 121-130

Kits K D, Jung M Y, Vierheilig J, Pjevac P, Sedlacek C J, Liu S, Herbold C, Stein L Y, Richter A, Wissel H, Brüggemann N, Wagner M, Daims $\mathrm{H}$ (2019). Low yield and abiotic origin of $\mathrm{N}_{2} \mathrm{O}$ formed by the complete nitrifier Nitrospira inopinata. Nature Communications, 10 (1): 1836
Kits K D, Sedlacek C J, Lebedeva E V, Han P, Bulaev A, Pjevac P, Daebeler A, Romano S, Albertsen M, Stein L Y, Daims H, Wagner M (2017). Kinetic analysis of a complete nitrifier reveals an oligotrophic lifestyle. Nature, 549(7671): 269-272

Knief C, Kolb S, Bodelier P L E, Lipski A, Dunfield P F (2006). The active methanotrophic community in hydromorphic soils changes in response to changing methane concentration. Environmental Microbiology, 8(2): 321-333

Koch H, van Kessel M A H J, Lücker S (2019). Complete nitrification: insights into the ecophysiology of comammox Nitrospira. Applied Microbiology and Biotechnology, 103(1): 177-189

Könneke M, Bernhard A E, de la Torre J R, Walker C B, Waterbury J B, Stahl D A (2005). Isolation of an autotrophic ammonia-oxidizing marine archaeon. Nature, 437(7058): 543-546

Kuypers M M M (2017). Microbiology: A fight for scraps of ammonia. Nature, 549(7671): 162-163

Kuypers M M M, Marchant H K, Kartal B (2018). The microbial nitrogen-cycling network. Nature Reviews. Microbiology, 16(5): 263-276

Lawson C E, Lücker S (2018). Complete ammonia oxidation: An important control on nitrification in engineered ecosystems? Current Opinion in Biotechnology, 50: 158-165

Liu S, Wang H, Chen L, Wang J, Zheng M, Liu S, Chen Q, Ni J (2020a). Comammox Nitrospira within the Yangtze River continuum: community, biogeography, and ecological drivers. The ISME Journal, 14(10): 2488-2504

Liu Z, Zhang C, Wei Q, Zhang S, Quan Z, Li M (2020b). Temperature and salinity drive comammox community composition in mangrove ecosystems across southeastern China. The Science of the total environment, 742: 140456

Lücker S, Wagner M, Maixner F, Pelletier E, Koch H, Vacherie B, Rattei T, Damsté J S, Spieck E, Le Paslier D, Daims H (2010). A Nitrospira metagenome illuminates the physiology and evolution of globally important nitrite-oxidizing bacteria. Proceedings of the National Academy of Sciences of the United States of America, 107(30): 13479-13484

Palomo A, Pedersen A G, Fowler S J, Dechesne A, Sicheritz-Pontén T, Smets B F (2018). Comparative genomics sheds light on niche differentiation and the evolutionary history of comammox Nitrospira. The ISME Journal, 12(7): 1779-1793

Pinto A J, Marcus D N, Ijaz U Z, Bautista-de Lose Santos Q M, Dick G J, Raskin L (2015). Metagenomic evidence for the presence of comammox Nitrospira-like bacteria in a drinking water system. MSphere, 1(1): e00054-e15

Pjevac P, Schauberger C, Poghosyan L, Herbold C W, van Kessel M A H J, Daebeler A, Steinberger M, Jetten M S M, Lücker S, Wagner M, Daims H (2017). AmoA-targeted polymerase chain reaction primers for the specific detection and quantification of comammox Nitrospira in the environment. Frontiers in Microbiology, 8: 1508

Roots P, Wang Y, Rosenthal A F, Griffin J S, Sabba F, Petrovich M, Yang F, Kozak J A, Zhang H, Wells G F (2019). Comammox Nitrospira are the dominant ammonia oxidizers in a mainstream low dissolved oxygen nitrification reactor. Water Research, 157: 396-405

Sakoula D, Koch H, Frank J, Jetten M S M, van Kessel M A H J, Lücker S (2021). Enrichment and physiological characterization of a novel comammox Nitrospira indicates ammonium inhibition of complete 
nitrification. The ISME journal, 15(4): 1010-1024

Sato Y, Tanaka E, Hori T, Futamata H, Murofushi K, Takagi H, Akachi T, Miwa T, Inaba T, Aoyagi T, Habe H (2021). Efficient conversion of organic nitrogenous wastewater to nitrate solution driven by comammox Nitrospira. Water Research, 197: 117088

Shao Y H, Wu J H (2021). comammox Nitrospira species dominate in an efficient partial nitrification-anammox bioreactor for treating ammonium at low loadings. Environmental Science \& Technology, 55(3): 2087-2098

Shen Y C, Hu Y N, Shaw G C (2016). Expressions of alkaline phosphatase genes during phosphate starvation are under positive influences of multiple cell wall hydrolase genes in Bacillus subtilis. The Journal of General and Applied Microbiology, 62(2): 106-109

Shi Y, Jiang Y Y, Wang S Y, Wang X M, Zhu G B (2020). Biogeographic distribution of comammox bacteria in diverse terrestrial habitats. Science of the Total Environment, 717: 137257

Steenbergh A K, Meima M M, Kamst M, Bodelier P L E (2010). Biphasic kinetics of a methanotrophic community is a combination of growth and increased activity per cell. FEMS Microbiology Ecology, 71(1): 12-22

Sun D Y, Tang X F, Zhao M Y, Zhang Z X, Hou L J, Liu M, Wang B Z, Klümper U, Han P (2020). Distribution and diversity of comammox Nitrospira in coastal wetlands of China. Frontiers in Microbiology, 11: 589268

Sun D Y, Zhao M Y, Tang X F, Liu M, Hou L J, Zhao Q, Li J, Gu J D, Han P (2021). Niche adaptation strategies of different clades of comammox Nitrospira in the Yangtze Estuary. International Biodeterioration \& Biodegradation, 164: 105286

Takahashi Y, Fujitani H, Hirono Y, Tago K, Wang Y, Hayatsu M, Tsuneda S (2020). Enrichment of comammox and nitrite-oxidizing Nitrospira from acidic soils. Frontiers in Microbiology, 11: 1737

Tatari K, Musovic S, Gülay A, Dechesne A, Albrechtsen H, Smets B F (2017). Density and distribution of nitrifying guilds in rapid sand filters for drinking water production: Dominance of Nitrospira spp. Water Research, 127: 239-248

Teske A, Alm E, Regan J M, Toze S, Rittmann B E, Stahl D A (1994). Evolutionary relationships among ammonia- and nitrite-oxidizing bacteria. Journal of Bacteriology, 176(21): 6623-6630

Van Kessel M A H J, Speth D R, Albertsen M, Nielsen P H, Op den Camp H J M, Kartal B, Jetten M S M, Lücker S (2015). Complete nitrification by a single microorganism. Nature, 528(7583): 555-559

Vigliotta G, Nutricati E, Carata E, Tredici S M, De Stefano M, Pontieri P, Massardo D R, Prati M V, De Bellis L, Alifano P (2007). Clonothrix fusca Roze 1896, a filamentous, sheathed, methanotrophic $\gamma$ proteobacterium. Applied and Environmental Microbiology, 73 (11): 3556-3565

Wang M Y, Huang G H, Zhao Z R, Dang C Y, Liu W, Zheng M S (2018). Newly designed primer pair revealed dominant and diverse comammox amoA gene in full-scale wastewater treatment plants. Bioresource Technology, 270: 580-587
Wang S Y, Pi Y L, Jiang Y Y, Pan H W, Wang X X, Wang X M, Zhou Z M, Zhu G B (2019). Nitrate reduction in the reed rhizosphere of a riparian zone: From functional genes to activity and contribution. Environmental Research, 180(6): 108867

Wang Y, Ma L, Mao Y, Jiang X, Xia Y, Yu K, Li B, Zhang T (2017). Comammox in drinking water systems. Water Research, 116: 332341

Wang Y L, Zhao R X, Liu L, Li B, Zhang T (2021). Selective enrichment of comammox from activated sludge using antibiotics. Water Research, 197: 117087

Wang Z, Zhang L, Zhang F Z, Jiang H, Ren S, Wang W, Peng Y Z (2020). Nitrite accumulation in comammox-dominated nitrificationdenitrification reactors: Effects of DO concentration and hydroxylamine addition. Journal of Hazardous Materials, 384: 121375

Winkler M K, Bassin J P, Kleerebezem R, Sorokin D Y, van Loosdrecht M C (2012). Unravelling the reasons for disproportion in the ratio of AOB and NOB in aerobic granular sludge. Applied Microbiology and Biotechnology, 94(6): 1657-1666

Winogradsky S (1890). The morphology of the contributions of nitrification system. Archives of Biological Sciences, 4: 257-275

Xia F, Wang J G, Zhu T, Zou B, Rhee S K, Quan Z X (2018). Ubiquity and diversity of complete ammonia oxidizers (comammox). Applied and Environmental Microbiology, 84(24): e01390-e18

Xu S Y, Wu X L, Lu H J (2021). Overlooked nitrogen-cycling microorganisms in biological wastewater treatment. Frontiers of Environmental Science \& Engineering, 15(6): 133

Xu Y F, Lu J, Wang Y C, Liu G L, Wan X Q, Hua Y M, Zhu D W, Zhao J W (2020). Diversity and abundance of comammox bacteria in the sediments of an urban lake. Journal of Applied Microbiology, 128(6): $1647-1657$

Yu C, Hou L, Zheng Y, Liu M, Yin G, Gao J, Liu C, Chang Y, Han P (2018). Evidence for complete nitrification in enrichment culture of tidal sediments and diversity analysis of clade a comammox Nitrospira in natural environments. Applied Microbiology and Biotechnology, 102(21): 9363-9377

Zeng W, Zhang L M, Wang A Q, Zhang J, Peng Y Z, Duan J L (2015). Community structures and population dynamics of nitrifying bacteria in activated sludges of wastewater treatment plants. China Environmental Science, 35(11): 3257-3265 (in Chinese)

Zhao Y X, Hu J J, Yang W L, Wang J Q, Jia Z J, Zheng P, Hu B L (2021). The long-term effects of using nitrite and urea on the enrichment of comammox bacteria. Science of the Total Environment, 755(Pt 2): 142580

Zhao Z, Huang G, He S, Zhou N, Wang M, Dang C, Wang J, Zheng M (2019). Abundance and community composition of comammox bacteria in different ecosystems by a universal primer set. Science of the Total Environment, 691: 146-155

Zhou X, Li B, Wei J, Ye Y, Xu J, Chen L, Lu C (2021). Temperature influenced the comammox community composition in drinking water and wastewater treatment plants. Microbial Ecology, 82(4): 870-884 